\title{
Eigenstate thermalization hypothesis and modular invariance of two-dimensional conformal field theories
}

\author{
Yasuaki Hikida, ${ }^{1}$ Yuya Kusuki, ${ }^{1}$ and Tadashi Takayanagi ${ }^{1,2}$ \\ ${ }^{1}$ Center for Gravitational Physics, Yukawa Institute for Theoretical Physics (YITP), Kyoto University, \\ Kitashirakawa Oiwakecho, Sakyo-ku, Kyoto 606-8502, Japan \\ ${ }^{2}$ Kavli Institute for the Physics and Mathematics of the Universe, University of Tokyo, \\ Kashiwano-ha, Kashiwa, Chiba 277-8582, Japan
}

(Received 7 May 2018; published 3 July 2018)

\begin{abstract}
We study properties of heavy-light-heavy three-point functions in two-dimensional conformal field theories by using the modular invariance of two-point functions on a torus. We show that the result is nontrivially consistent with the condition of eigenstate thermalization hypothesis. We also study the openclosed duality of cylinder amplitudes and derive behaviors of disk one-point functions.
\end{abstract}

DOI: $10.1103 /$ PhysRevD.98.026003

\section{INTRODUCTION}

Two-dimensional conformal field theories (2D CFTs) have provided us an ideal factory of new insights on dynamical properties in quantum field theories. This is owing highly to the strong constraints imposed by their infinite dimensional conformal symmetries [1]. One wellknown highlight is the Cardy formula

$$
D(E) \sim e^{4 \pi \sqrt{\frac{C E}{12}}}
$$

which offers a universal formula for the degeneracy $D(E)$ of highly excited states (with energy $E \gg c$ ) for general unitary 2D CFTs with the central charge $c$ [2]. This formula is derived from the modular invariance of torus amplitude. In the presence of boundaries, we can employ the openclosed duality to constrain the behaviors of boundary states [3] in boundary conformal field theories [4]. Moreover, the conformal bootstrap for correlation functions leads to another strong constraints on the properties of CFTs, which has successfully been applied not only in two-dimensions but also in higher-dimensions [5,6].

Recently, there have been very interesting progresses on the properties of three-point functions in 2D CFTs. The modular invariance of a torus one-point function leads to a universal formula for the diagonal part of heavy-lightheavy three-point functions [7]. The genus two modular invariance gives another constraints on the behavior of

Published by the American Physical Society under the terms of the Creative Commons Attribution 4.0 International license. Further distribution of this work must maintain attribution to the author(s) and the published article's title, journal citation, and DOI. Funded by SCOAP ${ }^{3}$. heavy-heavy-heavy three-point functions [8], see also $[9,10]$. The behaviors of heavy-light-light three-point functions have been worked out in [11,12]. Refer to [13-20] for other aspects of recent developments on constraints in 2D CFTs. For generic CFTs, heavy operators (or equally states) are those with energy (or equally conformal dimensions) larger than the central charge $c$. In particular, for holographic CFTs, which are strongly coupled and have a large central charge $c$, we can say operators are heavy already when its energy is larger than $c / 6$. Operators with energies much smaller than those of the heavy ones are simply called light.

Motivated by these developments, the purpose of this article is to explore more of such universal properties through the modular invariance of two-point functions on a torus [21-23] and the open-closed duality [4]. As we will explain later, the former analysis leads to interesting constraints for the off diagonal part of heavy-light-heavy three-point functions. The results satisfy the condition of ETH (eigenstate thermalization hypothesis) [24-26], which is a well-known criterion for a closed quantum system to become chaotic such that it gets thermalized. We will also generalize this argument to multipoint functions on a torus. The latter analysis provides new universal properties on the one-point functions on a disk or equally the coefficients of boundary states. Our analysis below focuses only on the exponential contributions neglecting the polynomial factor of the energy of relevant states, which is denoted by the symbol $\sim$. Refer to [27-33] for earlier arguments on ETH in CFTs.

This article is organized as follows: in Sec. II, we study the modular invariance of two-point functions on a torus and derive a property of three-point functions. After briefly introducing the ETH, we show that the result satisfies the condition of ETH. In Sec. III, we study the open-closed 
duality and derive constraints on the coefficients of boundary states. We also give a holographic interpretation. In Sec. IV, we summarize our conclusions and discuss future problems.

\section{MODULAR INVARIANCE ON TORUS AND ETH}

Consider a two-point function $\langle O(t, \phi) O(0,0)\rangle$ of a primary operator $O$ on a torus in a given 2D CFT. We choose $O$ such that its spin is vanishing. Its (chiral) conformal dimensions are given by $\Delta_{O}=\bar{\Delta}_{O}$, and its energy reads $E_{O}=2 \Delta_{O}$. The Euclidean time coordinate $t$ and space coordinate $\phi$ are periodic such that $t \sim t+\beta$ and $\phi \sim \phi+2 \pi$ on this torus.

We would like to study the constraint imposed by the modular invariance (where we follow the convention in [23]). Until Sec. II. B., we assume generic 2D CFTs below. We will discuss how the results are improved for holographic CFTs in Sec. II. C. We interpret our results in the light of ETH in Sec. II. 4.

By setting $\phi=0$ for simplicity, the modular invariant relation $\beta \leftrightarrow(2 \pi)^{2} / \beta$ for the torus two point function $\langle O(t, 0) O(0,0)\rangle$ is written as

$$
\begin{aligned}
& e^{\frac{\beta c}{12}} \sum_{A} \sum_{B}|\langle A|O| B\rangle|^{2} e^{-(\beta-t) E_{A}-t E_{B}} \\
& =e^{\frac{(2 \pi)^{2} c}{12 \beta}} \cdot\left(\frac{2 \pi}{\beta}\right)^{2 E_{O}} \sum_{A} \sum_{B} e^{-\frac{(2 \pi)^{2} E_{A}}{\beta}} e^{\frac{2 \pi i I_{A B} t}{\beta}}|\langle A|O| B\rangle|^{2},
\end{aligned}
$$

where $A$ and $B$ label all states in the CFT including both primaries and descendants; we set $E_{A}=\Delta_{A}+\bar{\Delta}_{A}$ as the energy of the state $|A\rangle$ and $J_{A B}$ as the difference of the spins of $|A\rangle$ and $|B\rangle$. The matrix element $\langle A|O| B\rangle$ is equal to the three-point function $\langle A(\infty) O(1) B(0)\rangle$.

Below we would like to study implications of (2) in the high temperature limit $\beta \rightarrow 0$ with $t / \beta$ kept finite and nonvanishing. In this case, we do not need to worry about the divergence due to the coincidence of two operators (i.e., $t=0$ ). In this limit we can set $A$ to be the vacuum. By using the Cardy formula (1) for the degeneracy of high energy states $E_{A, B} \gg c$, we can estimate both sides of the modular invariance relation (2) for the torus two point function $\langle O(t, 0) O(0,0)\rangle$ as follows:

$$
\begin{aligned}
& \int d E_{A} d E_{B} e^{4 \pi \sqrt{\frac{c}{12} E_{A}}} e^{4 \pi \sqrt{\frac{c}{12} E_{B}}} \overline{\left.\langle A|O| B\rangle\right|^{2}} e^{-(\beta-t) E_{A}-t E_{B}} \\
& \sim e^{\frac{\pi^{2} c}{3 \beta}} \cdot\left(\frac{2 \pi}{\beta}\right)^{2 \Delta_{O}} .
\end{aligned}
$$

Here, the average is over all states $A$ and $B$ of fixed dimensions $E_{A}$ and $E_{B}$. Since in general $E_{A}$ and $E_{B}$ can be arbitrarily large in the limit $\beta \rightarrow 0$, the above relation (3) provides a constraint on the mean squared of heavy-light- heavy three-point functions $\overline{|\langle A|O| B\rangle|^{2}}$ as a function of $E_{A}$ and $E_{B}$. In the analysis below we only focus on the exponential contributions neglecting the polynomial factor of the energy $E_{A}$ and $E_{B}$.

When $t / \beta$ is very small, the dominant contributions of the left-hand side of (3) come from the states with $E_{B} \gg E_{A}$. Also if we strictly set $t \rightarrow 0$, then the two-point function should have a power divergence $\sim t^{-2 E_{O}}$. Thus we find that the summation over $B$ should marginally converge when $t / \beta$ is very small, and this leads to the following estimation of the exponential suppression of the three-point functions when $E_{B} \gg E_{A}$ :

$$
\overline{\left|\langle A|O| B\rangle_{\mathrm{off}}\right|^{2}} \sim e^{-4 \pi \sqrt{\frac{c}{12} E_{B}}} .
$$

We would also like to comment that if we keep $\beta$ finite and take the limit $t \rightarrow 0$, then the two-point function behaves as $\langle O(t, 0) O(0,0)\rangle \simeq t^{-2 E_{O}} \cdot Z(\beta)$, where $Z(\beta)$ is the vacuum partition function. This relation is equivalent to the conformal bootstrap constraint for four-point functions studied in $[11,12]$.

\section{A. Constraints on 3pt functions}

Now we would like to estimate the square of the off diagonal part of the three-point function $\overline{\left|\langle A|O| B\rangle_{\text {off }}\right|^{2}}$ from the modular invariance (3).

Let us introduce the parameter $s$ instead of $t$,

$$
\beta-t=\frac{\beta+s}{2}, \quad t=\frac{\beta-s}{2},
$$

where $-\beta \leq s \leq \beta$. Moreover, we parametrize $E_{A}$ and $E_{B}$ by the "boost coordinate" $(\rho, \theta)$ as follows:

$$
E_{A}=\rho e^{\theta}, \quad E_{B}=\rho e^{-\theta},
$$

where $-\infty<\theta<\infty$ and $0<\rho<\infty$.

We assume the following behavior of the three-point function square:

$$
\overline{\left|\langle A|O| B\rangle_{\text {off }}\right|^{2}} \sim e^{-4 \pi \sqrt{\frac{c \rho}{12}} f(\theta)},
$$

where $f(\theta)$ is an unknown function, which we want to determine from the modular property (3) below. This form is expected from the requirement that the degeneracy given by the Cardy formula should be canceled in the integral in (3) and is justified in our analysis below. 
The left-hand side of (3) is estimated by the integral,

$$
\begin{aligned}
\int & \rho d \rho d \theta e^{I(\rho, \theta)}, \\
I(\rho, \theta) \equiv & 4 \pi \sqrt{\frac{c E_{A}}{12}}+4 \pi \sqrt{\frac{c E_{B}}{12}}-4 \pi \sqrt{\frac{c \rho}{12}} f(\theta) \\
& -(\beta-t) E_{A}-t E_{B} \\
= & 8 \pi \sqrt{\frac{c \rho}{12}} \cosh \left(\frac{\theta}{2}\right)-4 \pi \sqrt{\frac{c \rho}{12}} f(\theta)-\beta \rho \cosh \theta \\
& -s \rho \sinh \theta .
\end{aligned}
$$

Now we apply the saddle point approximation with respect to the integral of $\rho$. This leads to the following relation by solving $\partial_{\rho} I=0$ :

$$
\sqrt{\rho}=2 \pi \sqrt{\frac{c}{12}}\left(\frac{2 \cosh \left(\frac{\theta}{2}\right)-f(\theta)}{\beta \cosh \theta+s \sinh \theta}\right) .
$$

By substituting this and assuming $f(\theta)<2 \cosh \left(\frac{\theta}{2}\right), I(\rho, \theta)$ is simplified,

$$
I(\rho, \theta)=\frac{\pi^{2} c}{3} \cdot \frac{\left(2 \cosh \left(\frac{\theta}{2}\right)-f(\theta)\right)^{2}}{\beta \cosh \theta+s \sinh \theta} .
$$

Note that if $f(\theta)>2 \cosh \left(\frac{\theta}{2}\right)$, then there is no saddle point (9) and the related contributions can be neglected.

Now we would like to impose the modular invariance (3). This means the following equivalence in the limit $\beta \rightarrow 0$ and $s / \beta=$ finite:

$$
\int_{-\infty}^{\infty} d \theta e^{\frac{\pi^{2} c}{3} \cdot \frac{\left(2 \cosh \left(\frac{\theta}{2}\right)-f(\theta)\right)^{2}}{\beta \cosh \theta+\sinh \theta}} \sim e^{\frac{\pi^{2} c}{3 \beta}} .
$$

This approximated equality should be true for any values of the ratio $s / \beta$ (remember $s$ takes the values such that $|s / \beta| \leq 1)$. The important point is that the right-hand side does not depend on the parameter $s$, and this is possible only if the contribution from the integral of $\theta$ is localized around $\theta=0$. This requires (i) $f(0) \simeq 1$ and (ii) the integral for $\theta \neq 0$ can be negligible, which is equal to the inequality,

$$
\frac{\left(2 \cosh \left(\frac{\theta}{2}\right)-f(\theta)\right)^{2}}{\beta \cosh \theta+s \sinh \theta}<\frac{1}{\beta},
$$

for any $|s| \leq \beta$. This inequality (12) is equivalent to

$$
f(\theta) \geq 2 \cosh \left(\frac{\theta}{2}\right)-e^{-\frac{|\theta|}{2}}=e^{\frac{|\theta|}{2}},
$$

where we used $f(\theta)<2 \cosh \left(\frac{\theta}{2}\right)$. Moreover, the previous argument which derives (4), tells us $f(\theta) \simeq e^{\frac{|\theta|}{2}}$ in the limit $|\theta| \rightarrow \infty$.
In summary, we find the following behavior:

$$
\begin{array}{cl}
\text { If } E_{A} \simeq E_{B} \gg c, & \overline{\left|\langle A|O| B\rangle_{\mathrm{off}}\right|^{2}} \sim e^{-\frac{S\left(E_{A}\right)+S\left(E_{B}\right)}{2}}, \\
\text { If } c \ll E_{A} \ll E_{B}, & \overline{\left|\langle A|O| B\rangle_{\mathrm{off}}\right|^{2}} \sim e^{-S\left(E_{B}\right)},
\end{array}
$$

For generic values of $E_{A, B} \gg c$,

$$
\overline{\left|\langle A|O| B\rangle_{\text {off }}\right|^{2}} \lesssim \operatorname{Min}\left[e^{-S\left(E_{A}\right)}, e^{-S\left(E_{B}\right)}\right],
$$

where $S(E)=4 \pi \sqrt{\frac{c E}{12}}$ is the entropy for states with the energy $E$.

In the above analysis, we ignored contributions from the diagonal part $\langle A|O| B\rangle_{\text {diag }}=\delta_{A B} \cdot\langle A|O| A\rangle$. The averaged diagonal three-point function is found in [7]

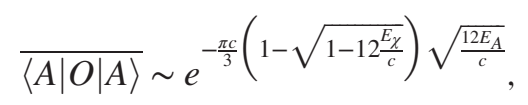

where $E_{\chi}$ is the energy of the lowest dimensional state $|\chi\rangle$ which satisfies $\langle\chi|O| \chi\rangle \neq 0$. By explicitly substituting this to (3), we find that the diagonal ones are not dominant over the off diagonal ones, and this justifies the above analysis.

Note that we can actually show that in the limit $E_{B} \rightarrow \infty$ with $E_{B}-E_{A}$ fixed, the two-point conformal blocks on a torus take just the form of a torus character [20]. Therefore, the three-point function of primary states can be given by the same formula (14) after just a shift $c \rightarrow c-1$ up to a constant factor as in [7]. However, in the case $E_{A} \ll E_{B}$, the contribution from the two-point block on a torus is nontrivial, and therefore the above result does not contradict with the results in $[11,12]$.

\section{B. H-L-L 3pt functions from conformal bootstrap}

Let us focus on the case where only either $E_{A}$ or $E_{B}$ is heavy, i.e. the heavy-light-light three-point function. Our previous argument based on the modular invariance derives the behavior (4) as we have explained. Here we would like to point out that this result for the heavy-light-light threepoint function can also be found from a simple argument of conformal bootstrap. First note that the four-point function of primary operators $O_{1}$ and $O_{2}$ can be expanded in terms of all states $A$ in a given 2D CFT,

$$
\begin{aligned}
& \left\langle O_{1}(0) O_{2}(x, \bar{x}) O_{2}(1) O_{1}(\infty)\right\rangle \\
& \quad=|x|^{-E_{O_{1}}-E_{O_{2}}} \sum_{A}\left|C_{O_{1} O_{2} A}\right|^{2} x^{\Delta_{A}} \bar{x}^{\bar{\Delta}_{A}},
\end{aligned}
$$

where $C_{O_{1} O_{2} A}=\left\langle O_{1}\left|O_{2}\right| A\right\rangle$. The bootstrap relation in the limit $x \rightarrow 1$ leads to the behavior

$$
\left\langle O_{1}(0) O_{2}(x, \bar{x}) O_{2}(1) O_{1}(\infty)\right\rangle \simeq|1-x|^{-2 E_{O_{2}}} .
$$

Therefore we obtain the following relation in the limit $x=\bar{x} \rightarrow 1$ : 


$$
\int d E_{A} D\left(E_{A}\right) \overline{\left|C_{O_{1} O_{2} A}\right|^{2}} x^{E_{A}} \sim|1-x|^{-2 E_{O_{2}}},
$$

where the density of state reads $D\left(E_{A}\right) \sim e^{2 \pi \sqrt{\frac{c E_{A}}{3}}}$. It is straightforward to see that this leads to the behavior,

$$
\overline{\left|C_{O_{1} O_{2} A}\right|^{2}} \sim e^{-4 \pi \sqrt{\frac{c E_{A}}{12}}}
$$

which indeed reproduces (4).

\section{Holographic CFTs}

So far our arguments assumed generic unitary CFTs in two-dimensions, where we have shown the exponential suppressions of the off diagonal three-point functions for the energies much larger than the central charge $E_{A}$, $E_{B} \gg c$. However, if we consider the special class of CFTs, called holographic CFTs, this energy condition is relaxed as we will explain below. The holographic CFTs are characterized by the large degrees of freedom and strong interactions so that they have classical holographic duals. In two-dimensional CFTs, these conditions are equivalent to the large central charge $c \gg 1$ and the sparse spectrum. Under these conditions, the Cardy formula ${ }^{1} D(E) \sim$ $e^{4 \pi \sqrt{\frac{c}{12}\left(E-\frac{c}{12}\right)}}$ holds for any states with the energy $E=$ $\Delta+\bar{\Delta} \geq \frac{c}{6}$ as proved in [15], which is equivalent to a sharp confinement/deconfinement phase transition of the partition function at $\beta=2 \pi$. Therefore we can employ the Cardy formula even for such relatively low energy states.

Let us ask if we can apply the previous argument in Sec. II A to such states $E_{A, B}=O(c)$. The crucial point is the validity of the saddle point approximation (9). Since the dominant contribution is localized at $\theta=0$, we find that the saddle point is located at $E_{A, B}-\frac{c}{12} \simeq \rho=\left(\frac{2 \pi}{\beta}\right)^{2} \cdot \frac{c}{12}$. Since we consider the low temperature phase for the right-hand side of the basic relation (2), we have the condition $\beta<2 \pi$. From this, we obtain $E_{A, B} \geq \frac{c}{6}$. In this way, we can conclude that the exponential suppressions of the off diagonal threepoint functions (14), (15) and (16) occur in 2D holographic CFTs when $E_{A, B} \geq \frac{c}{6}$, which allow much lower energy regions compared with the condition of the same suppressions for generic CFTs, i.e., $E_{A, B} \gg c$.

\section{Comparison with ETH}

The ETH (eigenstate thermalization hypothesis) [24-26], which we will briefly review below, is formulated for the matrix elements of observables $O$ in the basis $|n\rangle$ for the eigenstates of Hamiltonian $H$,

\footnotetext{
${ }^{1}$ Note that in (1) we assumed $E \gg c$, and thus we made the approximation $E-\frac{c}{12} \simeq E$. In this section, we choose $E$ and $c$ as both equally large, and we cannot allow this approximation.
}

$$
\langle n|O| m\rangle=f_{O}\left(E_{n}\right) \delta_{n m}+e^{-\frac{S(E)}{2}} g_{O}\left(E_{n}, E_{m}\right) R_{n m},
$$

where $S(E)$ is the averaged entropy at the energy $E=\frac{E_{n}+E_{m}}{2}$. The matrix $R_{n m}$ is a random Hermitian matrix with zero mean and unit variance. Ideally, we expect

$$
\left\langle\left\langle R_{k l} R_{m n}\right\rangle\right\rangle=\delta_{l, m} \delta_{k, n},
$$

where $\langle\langle\cdots\rangle\rangle$ denotes the random average. ${ }^{2}$ The functions $f_{O}\left(E_{n}\right)$ and $g_{O}\left(E_{n}, E_{m}\right)$ are smooth functions of the energies $E_{n, m}$. This behavior (22) can be obtained by assuming that the eigenstates $|n\rangle$ are random states, i.e., if we choose a basis $|i\rangle$ such that the observable $O$ is diagonal $\langle i|O| j\rangle=\delta_{i j} O_{i}$, then the vectors $\left\{p_{i}^{n}\right\}$ defined by $|n\rangle=\sum_{i} p_{i}^{n}|i\rangle$ are random such that $\left\langle\left\langle p_{i}^{* n} p_{j}^{m}\right\rangle\right\rangle=\frac{1}{D} \delta_{i j} \delta_{n m}$, where $D$ is the dimension of Hilbert space.

The ETH is considered to be true in a closed quantum system with a quantum chaos. If we assume ETH, we can show the thermalization of the observable $O$ as follows. Consider the time evolution of expectation value $\langle O(t)\rangle$ for a quantum state $|\psi\rangle=\sum_{n} b_{n}|n\rangle$,

$$
\begin{aligned}
\langle O(t)\rangle & =\sum_{n, m}\langle n|O| m\rangle b_{n}^{*} b_{m} e^{i\left(E_{n}-E_{m}\right) t} \\
& =\sum_{n}\left|b_{n}\right|^{2}\langle n|O| n\rangle+\sum_{n \neq m} e^{i\left(E_{n}-E_{m}\right) t}\langle n|O| m\rangle b_{n}^{*} b_{m} .
\end{aligned}
$$

We say that $O$ is thermalized if the (some) time average of $\langle O(t)\rangle$ coincides with its microcanonical prediction. For this we need to show that (i) the time average $\bar{O}=\frac{1}{T} \int_{0}^{T} d t\langle O(t)\rangle$ only depends on the energy, and it should not depend on the details of the coefficient $b_{n}$, and moreover, (ii) the fluctuation is very small: $\frac{1}{T} \int_{0}^{T} d t\left\langle(O(t))^{2}\right\rangle-(\bar{O})^{2} \sim e^{-S(E)}$. Indeed, we can confirm that (i) is satisfied because the diagonal part of (22) only depends on the energy and that (ii) is satisfied because the off diagonal part is suppressed by the factor $e^{-S / 2}$.

In the ETH, we regard $O$ as a low energy operator and take the states $|n\rangle$ to be high energy states which are responsible for thermalizations. The ETH property (22) leads to the estimation when we take an appropriate average,

$$
\left\langle\left\langle|\langle n|O| m\rangle|^{2}\right\rangle\right\rangle \simeq e^{-S(E)}\left(g_{O}\left(E_{n}, E_{m}\right)\right)^{2} .
$$

\footnotetext{
${ }^{2}$ It has been pointed out that this random behavior is not exactly correct in generic chaotic quantum systems because $R_{n m}$ satisfy various constraints $[28,34]$. Our analysis for generic CFTs is not affected by this issue as we consider averaged quantities. For holographic CFTs, we expect this issue will be almost absent as the number of such kinematical constraints are much smaller than those of the total degrees of freedom.
} 
Indeed this exponentially suppressed behavior nicely agrees with our results (14), (15) and (16) for the off diagonal part of the heavy-light-heavy three-point functions, by identifying $A=n$ and $B=m$. In this way, the modular invariance in 2D CFTs nicely reproduces the ETH property for off diagonal three point functions. Note that for generic unitary 2D CFTs, these ETH behaviors for the averaged three point functions hold for the high energy states $E_{n}, E_{m} \gg c$ as we have seen in Sec. II A. In particular, for holographic CFTs, these properties hold even for relatively low energy states as long as the condition $E_{n}, E_{m} \geq \frac{c}{6}$ is satisfied.

Also note that the diagonal part (17), which is clearly a smooth function, gives the function $f_{O}(E)$ in (22). For holographic 2D CFTs, we expect $f_{O}(E)$ is indeed not exponentially suppressed because we normally have $E_{\chi}=$ $O(1)$ in (17) and take the large $c$ limit. For generic 2D CFTs, this ETH requirement of nonsuppressed $f_{O}(E)$ is not guaranteed.

\section{E. Torus multipoint functions and modular invariance}

The ETH (22) argues that the off diagonal three-point functions are not only exponentially suppressed but also are random valued. We would like to study their multipoint correlations to examine this property. For this, we consider the modular invariance of $N$-point functions on a torus $\left\langle O\left(t_{1}\right) O\left(t_{2}\right) \ldots O\left(t_{N}\right)\right\rangle$. For simplicity we choose $t_{i}=\frac{N-i}{N} \beta$ for $i=1,2, \ldots, N$. In the low temperature limit, this $N$-point function on a torus factorizes into the thermal partition function times the $N$-point function on a cylinder, where the latter does not have any exponentially growing factor. Similar to the $N=2$ case (2), the modular invariance of the torus $N$-point function in the limit $\beta \rightarrow 0$ leads to

$$
\begin{aligned}
& \quad \sum_{A_{1}, A_{2}, \ldots, A_{N}}\left\langle A_{1}|O| A_{2}\right\rangle\left\langle A_{2}|O| A_{3}\right\rangle \ldots\left\langle A_{N}|O| A_{1}\right\rangle \cdot e^{-\frac{\beta}{N}\left(E_{1}+E_{2}+\cdots+E_{N}\right)} \\
& \sim e^{\frac{\pi^{2} c}{3 \beta}}
\end{aligned}
$$

where we employed the estimation of the partition function $Z(1 / \beta) \sim e^{\frac{\pi^{2} c}{3 \beta}}$. The energies $E_{1}, E_{2}, \ldots$ are those of the states $\left|A_{1}\right\rangle,\left|A_{2}\right\rangle, \ldots$, respectively. By using the Cardy formula as before, we finally obtain from (26) the following average of the $N$ products of the three-point functions when all energies are the same $E_{1}=E_{2}=\cdots=E_{N} \equiv E$ :

$$
\overline{\left\langle A_{1}|O| A_{2}\right\rangle\left\langle A_{2}|O| A_{3}\right\rangle \ldots\left\langle A_{N}|O| A_{1}\right\rangle} \sim e^{(1-N) S(E)},
$$

where we do not take any summations over $A_{i}$; also $S(E)=4 \pi \sqrt{\frac{c E}{12}}$ is the entropy for states with the energy $E$ again.
Next we would like to compare this result with that obtained by assuming the ETH behavior (22), where $R_{n m}$ is a random matrix. When $N$ is even, we find

$$
\begin{aligned}
& \overline{\left\langle A_{1}|O| A_{2}\right\rangle\left\langle A_{2}|O| A_{3}\right\rangle \ldots\left\langle A_{N}|O| A_{1}\right\rangle} \\
& \quad \sim e^{-\frac{N}{2} S(E)} \overline{\left\langle\left\langle R_{A_{1} A_{2}} R_{A_{2} A_{3}} \ldots R_{A_{N} A_{1}}\right\rangle\right.} \sim e^{-(N-1) S(E) .}
\end{aligned}
$$

Here we evaluated the averaged random correlation function by the Wick contractions in the random average (23) as

$$
\begin{aligned}
& \overline{\left\langle R_{A_{1} A_{2}} R_{A_{2} A_{3}} \ldots R_{A_{N} A_{1}}\right\rangle} \\
& \quad=\overline{\delta_{A_{1} A_{3}} \delta_{A_{3} A_{5}} \ldots \delta_{A_{N-1} A_{1}}+\text { (permutation) }} \\
& \quad \sim e^{-\left(\frac{N}{2}-1\right) S(E)} .
\end{aligned}
$$

Remember that the average $\bar{M}$ in the above means that for all states with the energy $E$ i.e. $\bar{M}=e^{-N S(E)} \sum_{A_{1}, A_{2}, \ldots, A_{N}} M$. When $N$ is odd, we can obtain the same estimation (28) from the ETH ansatz (22) by replacing one of $e^{-S(E) / 2} R_{n m}$ with $\delta_{n, m}$ in the averaged random correlation function. In this way, the CFT result (27) reproduces the random matrix result (28) based on the ETH ansatz for these correlation functions. Note that again this analysis can applied to the high energy states $E \gg c$ for generic 2D CFTs and to the states $E \geq \frac{c}{6}$ for holographic CFTs.

\section{CONSTRAINTS FROM OPEN-CLOSED DUALITY}

Next we would like to study universal constraints for 2D CFTs with boundaries, where conformal boundary conditions are imposed. These theories are called as boundary conformal field theories. We are especially interested in properties of the one-point functions of primary operators on a disk. For this, it is useful to employ the description in terms of boundary states, which are closed string states for boundaries.

States which describe physical boundaries are called Cardy states $\left|B_{\alpha}\right\rangle$ [4] and are given by the linear combinations of Ishibashi states $\left|I_{k}\right\rangle$ as follows:

$$
\left|B_{\alpha}\right\rangle=\sum_{k} c_{k}^{\alpha}\left|I_{k}\right\rangle,
$$

where the label $k$ runs over those of primary states. The index $\alpha$ describes the types of boundary conditions.

The Ishibashi state $\left|I_{k}\right\rangle$ is constructed out of the primary state $|k\rangle$ and its descendants. It is given by the maximally entangled state between the left and right moving sectors,

$$
\left|I_{k}\right\rangle=\sum_{\vec{n}}|\vec{n}, k\rangle_{L}|\vec{n}, k\rangle_{R} .
$$

Here the states $|\vec{n}, k\rangle$ make the orthonormal basis of the descendants of the form 


$$
|\vec{n}, k\rangle=N_{\vec{n}, k}\left[\left(L_{-1}\right)^{n_{1}} \ldots\left(L_{-m}\right)^{n_{m}} \cdots+\cdots\right]|k\rangle,
$$

where $\left\{L_{m}\right\}$ are Virasoro generators and $N_{\vec{n}, k}$ are the overall constants to make the states orthonormal,

$$
\langle\vec{n}, k \mid \vec{m}, l\rangle=\delta_{k, l} \delta_{\vec{n}, \vec{m}} .
$$

\section{A. Duality for vacuum cylinder amplitudes}

The open-closed duality relation for vacuum cylinder amplitudes, so-called the Cardy condition [4], is written as

$$
\left\langle B_{\alpha}\left|e^{-s H_{C}}\right| B_{\beta}\right\rangle=\sum_{\gamma} N_{\alpha \beta}^{\gamma} \operatorname{Tr}_{\gamma}\left[e^{-2 \pi t H_{O}}\right],
$$

where $t$ and $s$ are related by

$$
t=\frac{\pi}{S} .
$$

Here $H_{C}$ and $H_{O}$ are the closed and open string Hamiltonians, respectively,

$$
H_{C}=L_{0}+\tilde{L}_{0}-\frac{c}{12}, \quad H_{O}=L_{0}-\frac{c}{24} .
$$

Moreover, $\gamma$ runs all primary states, and $N_{\alpha \beta}^{\gamma}$ is a positive integer which counts the number of open string sectors and is nonzero only when the corresponding OPE coefficient is nonvanishing.

\section{1. $t \rightarrow 0$ limit}

Let us first study a familiar limit $t \rightarrow 0$. Here we assume the limit of large central charge $c \gg 1$ so that the degeneracy of primary states is approximately given by the Cardy formula (1), with neglecting descendant contributions. We write the conformal dimension as $L_{0}=\Delta$ in the open string sector. In this limit, the duality condition (34) leads to

$$
\bar{c}_{0}^{\alpha} c_{0}^{\beta} \cdot e^{\frac{\pi c}{12 t}} \sim \int d \Delta D_{\mathrm{open}}(\Delta) e^{-2 \pi t\left(\Delta-\frac{c}{24}\right)},
$$

where $D_{\text {open }}(\Delta)$ is the density of state in an open string at energy $H_{O}=\Delta-\frac{c}{24}$. Here we have assumed that $c_{0}^{\alpha} \neq 0$ and $c_{0}^{\beta} \neq 0$ for boundary conditions $\alpha$ and $\beta$. These conditions are equivalent to those for vacuum disk amplitudes to be nonvanishing, and they are usually satisfied as they mean the nonzero tensions of D-branes. By using the saddle point approximation formula,

$$
\int d x e^{2 \pi \lambda \sqrt{\frac{c x}{6}}} e^{-2 s x} \sim e^{\frac{\pi^{2} c \lambda^{2}}{12 s}}
$$

we find the following estimation for $\Delta \gg c$ :

$$
D_{\text {open }}(\Delta) \sim e^{2 \pi \sqrt{\frac{c \Delta}{6}}}
$$

which gives the Cardy formula for open strings.

$$
\text { 2. } s \rightarrow 0 \text { limit }
$$

Now let us turn to the less familiar limit $s \rightarrow 0$. First we assume $\alpha=\beta$. We define the chiral conformal dimension as $L_{0}=\tilde{L}_{0}=\Delta_{k}$ for the primary state $|k\rangle$. In this case we get from (34),

$$
\int d \Delta_{k} \mid \overline{\left.c_{k}^{\alpha}\right|^{2}} e^{-\left(2 \Delta_{k}-\frac{c}{12}\right) s} D_{\text {closed }}\left(\Delta_{k}\right) \sim e^{\frac{\pi^{2} c}{12 s}} .
$$

Here the density of state (note that $L_{0}=\tilde{L}_{0}$ is imposed for boundary states) is given by the same as the chiral or open string Cardy formula for $\Delta_{k} \gg c$,

$$
D_{\text {closed }}\left(\Delta_{k}\right) \sim e^{2 \pi \sqrt{\frac{c \Delta_{k}}{6}}}
$$

which is a square root of the full Cardy formula (1). This leads to the following estimation:

$$
\overline{\left|c_{k}^{\alpha}\right|^{2}} \sim 1
$$

where an appropriate average over $k$ with fixed dimension $\Delta_{k}$ is taken.

Now we take the different boundary conditions $\alpha \neq \beta$. In this case, we have $N_{\alpha \beta}^{0}=0$, and thus there should be a gap in the open string spectrum. We call the minimum of the conformal dimension for the open string in the channel $\gamma$ as $\Delta_{\gamma}^{\min }$. Then, the relation (34) leads to

$$
\int d \Delta_{k} \overline{\bar{c}_{k}^{\alpha} c_{k}^{\beta}} e^{-\left(2 \Delta_{k}-\frac{c}{12}\right) s} D_{\text {closed }}\left(\Delta_{k}\right) \sim e^{\frac{\pi^{2}\left(c-24 \Delta_{\gamma}^{\min }\right)}{12 s}} .
$$

Thus we obtain the estimation

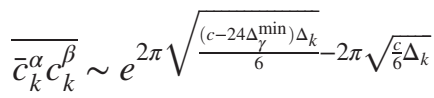

$$
\begin{aligned}
& =e^{-\frac{\pi c}{3}\left(1-\sqrt{1-\frac{24}{c} \Delta_{\gamma}^{\min }}\right) \sqrt{\frac{6}{c} \Delta_{k}}} .
\end{aligned}
$$

This can be understood as the correlations between the coefficients (or disk one-point functions) $c_{k}^{\alpha}$ and $c_{k}^{\beta}$. Compared with (42), we find that the correlation is reduced as the gap in the open strings between the two boundaries $\alpha$ and $\beta$ develops.

\section{Holographic interpretation}

Here we would like to give a holographic interpretation of the result (44), which is motivated by the argument in [7] 


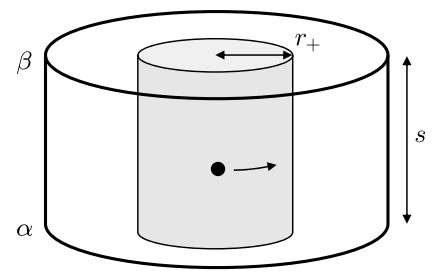

(a)

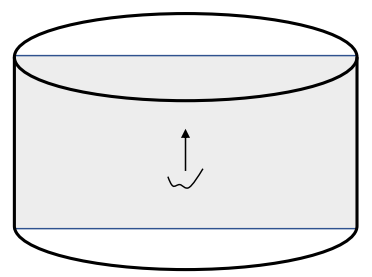

(b)
FIG. 1. (a) A closed string state at high energy and a light open string state are approximately described by the BTZ black hole and a perturbative scalar particle, respectively. (b) A bulk light particle dual to a boundary open string state can be regarded as a low energy excitation of a bulk open string on a brane.

for the diagonal three-point functions. ${ }^{3}$ We first consider the case with $1 \ll \Delta_{\gamma}^{\min } \ll \frac{c}{24}$, where the expression of (44) reduces to

$$
\overline{\bar{c}_{k}^{\alpha} c_{k}^{\beta}} \sim e^{-2 \pi \Delta_{\gamma}^{\min } \sqrt{\frac{24}{c} \Delta_{k}}}
$$

We then include the corrections as in (44) for $\Delta_{\gamma}^{\min }<\frac{c}{24}$.

We are interested in the exchange of closed strings with high energy $E_{k}=2 \Delta_{k} \gg c$ between the boundary states. The high energy state of closed string is dual to the BTZ black hole, whose horizon area is

$$
A=2 \pi r_{+}, \quad r_{+}=\sqrt{\frac{24 \Delta_{k}}{c}-1}
$$

For $\Delta_{\gamma}^{\min } \ll \frac{c}{24}$, the open string state with energy $\Delta_{\gamma}^{\min }$ can be described as a perturbative scalar particle with a mass $m_{\gamma}^{\min } \sim \Delta_{\gamma}^{\min }$. The leading order contribution comes from the scalar particle running around the BTZ black hole, see Fig. 1(a).

The other assumption $\Delta_{\gamma}^{\min } \gg 1$ implies that the contribution can be evaluated by the geodesic wrapping the black hole horizon as $\exp \left(-m_{\gamma}^{\min } A\right)$. Using $m_{\gamma}^{\min } \sim \Delta_{\gamma}^{\min }$ and the area of horizon (46) we reproduce (45) for $\Delta_{k} \gg c$.

Next we relax the condition of $\Delta_{\gamma}^{\min }$ as $\Delta_{\gamma}^{\min }<\frac{c}{24}$. In this case, we should take care of the backreaction of the particle since it would create a conical defect geometry. Let us consider a particle $\phi$ with a mass $m_{\phi}$ and its energy $E_{\phi}$ evaluated at the boundary of anti-de Sitter (AdS). The relation between them is given by [see (27) of [7] ]

$$
m_{\phi}=\frac{c}{6}\left(1-\sqrt{1-\frac{12 E_{\phi}}{c}}\right) .
$$

\footnotetext{
${ }^{3}$ A gravity dual interpretation is expected to be possible only for a holographic CFT with the condition of the large central charge and the sparse spectrum. However, as we will show below, we can reproduce the CFT result (44) without using the condition for the current case analogously for the Cardy formula (1).
}

However, we should be careful applying the formula to our setup since we are dealing with a bulk particle dual to a boundary open string state. The bulk particle is regarded as a low energy excitation of a bulk open string attached to a brane as in Fig. 1(b), see, e.g., [35,36] for AdS/BCFT. Since a pair of open strings create a closed string, we should set $2 m_{\gamma}^{\min }=m_{\phi}$ and $2 \Delta_{\gamma}^{\min }=E_{\phi}$. Therefore, we have

$$
m_{\gamma}^{\min }=\frac{c}{12}\left(1-\sqrt{1-\frac{24 \Delta_{\gamma}^{\min }}{c}}\right) .
$$

With this expression of $m_{\gamma}^{\min }$ and the area of the horizon (46), the contribution from the geodesic of the particle, $\exp \left(-m_{\gamma}^{\min } A\right)$, reproduces (44) for $\Delta_{k} \gg c$.

\section{B. Duality for 1pt function on cylinder}

Finally we would like to study the open-closed duality for a cylinder one-point function of a primary operator $O$,

$$
\left\langle B_{\alpha}\left|e^{-s_{1} H_{C}} O e^{-s_{2} H_{C}}\right| B_{\beta}\right\rangle=\sum_{\gamma} N_{\alpha \beta}^{\gamma} \operatorname{Tr}_{\gamma}\left[O e^{-2 \pi t H_{O}}\right],
$$

where $t=\frac{\pi}{s_{1}+s_{2}}$. Again we assume the limit of a large central charge $c \gg 1$.

In the limit $s_{1}, s_{2} \rightarrow 0$, this relation is expressed as follows:

$$
\begin{aligned}
\int & d \Delta_{k} d \Delta_{l} e^{2 \pi \sqrt{\frac{c \Delta_{k}}{6}}} e^{2 \pi \sqrt{\frac{c \Delta_{l}}{6}}} \overline{\langle k|O| l\rangle} \\
& \times e^{-\left(2 \Delta_{k}-c / 12\right) s_{1}} e^{-\left(2 \Delta_{l}-c / 12\right) s_{2}} \bar{c}_{k}^{\alpha} c_{l}^{\beta} \\
\simeq & N_{\alpha \beta}^{\gamma}\langle\gamma|O| \gamma\rangle_{\text {open }} \cdot e^{\frac{\pi^{2}}{12\left(s_{1}+s_{2}\right)}\left(c-24 \Delta_{\gamma O}^{\min }\right)},
\end{aligned}
$$

where $\gamma$ is the open string state with the smallest conformal dimension $L_{0}=\Delta_{\gamma O}^{\min }$, which satisfies $N_{\alpha \beta}^{\gamma} \neq 0$ and $\langle\gamma|O| \gamma\rangle_{\text {open }} \neq 0$. Note that by definition we have

$$
\Delta_{\gamma O}^{\min } \geq \Delta_{\gamma}^{\min }
$$

First we evaluate contributions from the diagonal part. We can employ the known formula (17) for the diagonal parts of three-point functions (with $L_{0}=\bar{L}_{0}=\Delta=E / 2$ ). Then, by using the saddle point formula and the previous formula (44), we can estimate the relation (50) and obtain the following relation:

$$
\sqrt{1-\frac{24}{c} \Delta_{\gamma O}^{\min }} \geq \sqrt{1-\frac{24}{c} \Delta_{\gamma}^{\min }}-2\left(1-\sqrt{1-\frac{24}{c} \Delta_{\chi}}\right) .
$$

The inequality should be saturated if the diagonal part gives the dominant contributions. Note that the inequality is consistent with (51). 
Next let us estimate contributions from the off diagonal parts of three-point functions. The open-closed duality tells us the relation

$$
\sum_{k, l} \overline{\langle k|O| l\rangle \bar{c}_{k}^{\alpha} c_{l}^{\beta}} e^{-2 \Delta_{k} s_{1}} e^{-2 \Delta_{l} s_{2}} \sim e^{\frac{\pi^{2}}{12\left(s_{1}+s_{2}\right)}\left(c-24 \Delta_{\gamma O}^{\min }\right)} .
$$

In particular, if we choose $s_{1}=s_{2}=s / 2$, we find

$$
\begin{aligned}
& \int d \Delta_{k} d \Delta_{l} e^{2 \pi \sqrt{\frac{c}{6} \Delta_{k}}+2 \pi \sqrt{\frac{c}{6} \Delta_{l}}} \cdot e^{-\left(\Delta_{k}+\Delta_{l}\right) s} \cdot \overline{\langle k|O| l\rangle \bar{c}_{k}^{\alpha} c_{l}^{\beta}} \\
& \sim e^{\frac{\pi^{2}}{12 s}\left(c-24 \Delta_{\gamma O}^{\min }\right)} .
\end{aligned}
$$

When $\Delta_{k} \simeq \Delta_{l}$, this relation leads to the behavior,

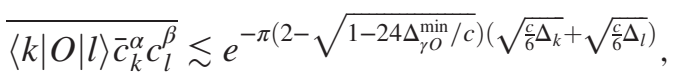

where the inequality is saturated when the off diagonal contributions are dominant. In other words, either (52) or (55) should be saturated in order for the open-closed duality relation (49) to be satisfied.

\section{CONCLUSIONS}

In this article, we studied implications of modular dualities in 2D CFTs. In particular, we analyzed the modular invariance of two-point functions on a torus and the openclosed duality of cylinder amplitudes with the input of density of high energy states given by the Cardy formula.

The modular invariance of two-point functions leads to nontrivial constraints on the behavior of three-point functions where two of the operators are heavy, whose dimensions are much larger than the central charge, and the other is much lighter than them. We found that the off diagonal part of three-point functions, for which the case with two different heavy states, is exponentially suppressed by the entropy as $\sim e^{-\frac{S(E)}{2}}$ under an appropriate average. Interestingly, this nontrivially satisfies the important condition required for the ETH. This implies that high energy states in generic 2D CFTs have the crucial property necessary for thermalizations. To see more details of how much a given 2D CFT is chaotic, we need to understand the properties of the matrix $R_{n m}$ in (22). For truly chaotic CFTs, we expect that $R_{n m}$ becomes a random matrix. Even though we leave the full studies of the random matrix property in 2D CFTs for a future problem, we found some evidence for this by studying a class of multipoint correlations of the off diagonal three-point functions.

It is natural to expect that for integrable CFTs, only with particular choices of $n$ and $m$, this matrix takes nontrivial values, and thus they do not satisfy ETH. In our analysis of modular invariance for general two-dimensional CFTs, we were able to show the suppression by the factor $e^{-S(E) / 2}$ of averaged three point functions only at very high energies $E \gg c$. On the other hand, we expect that large central charge CFTs with large spectrum gaps, namely holographic CFTs [15] satisfy ETH as they are expected to be maximally chaotic. Indeed, we found that for $2 \mathrm{D}$ holographic CFTs, both the exponential suppressions and the expected behavior for the multipoint correlations of the three-point functions, occur even for relatively low energy states $E \geq \frac{c}{6}$. These reinforce the chaotic properties for holographic CFTs. It will be an intriguing future problem to examine more closely the randomness of the matrix $R_{m n}$ for various 2D CFTs. The constraints for three-point functions also come from the conformal bootstrap. Recently, an interesting transition phenomenon for conformal blocks, depending on whether the conformal dimensions are larger than $c / 32$ or not, was observed in [12,37]. It would be interesting to consider its implication in terms of the behavior of three-point functions.

The open-closed duality for vacuum cylinder amplitudes turned out to predict interesting behaviors of disk one-point functions (or equally the coefficients of boundary states) for various conformal boundary conditions. We found that as the mass gap in open stings between two boundaries gets larger, the correlation between the two disk one-point functions for the boundary conditions is reduced. We gave a holographic explanation for this result. We also studied implications of the open-closed duality for cylinder onepoint functions. This leads to interesting constraints on the average of a three-point function times two disk one-point functions. It would be interesting to look at explicit examples of boundary states in solvable CFTs, such as various orbifold CFTs, to see how the chaos is related to these properties of boundary states.

\section{ACKNOWLEDGMENTS}

We thank Kanato Goto and Tokiro Numasawa very much for useful discussions. We are also grateful to Chen-Te Ma and Anatoly Dymarsky for valuable comments. T T would also like to thank Stanford Institute for Theoretical Physics for their hospitality where this work was completed. Y H and $\mathrm{T} T$ are supported by JSPS Grant-in-Aid for Scientific Research (A) Grant No. 16H02182. Y K received support from a JSPS fellowship. TT is also supported by the Simons Foundation through the "It from Qubit" Collaboration and by World Premier International Research Center Initiative (WPI Initiative) from the Japan Ministry of Education, Culture, Sports, Science and Technology (MEXT).

Note added.-When we were writing up this article, we noticed the interesting preprints $[38,39]$ on the arXiv, which have substantial overlaps with the Sec. II of our present one. 
[1] A. A. Belavin, A. M. Polyakov, and A. B. Zamolodchikov, Infinite conformal symmetry in two-dimensional quantum field theory, Nucl. Phys. B241, 333 (1984).

[2] J. L. Cardy, Operator content of two-dimensional conformally invariant theories, Nucl. Phys. B270, 186 (1986).

[3] N. Ishibashi, The boundary and crosscap states in conformal field theories, Mod. Phys. Lett. A 04, 251 (1989).

[4] J. L. Cardy, Boundary conditions, fusion rules and the Verlinde formula, Nucl. Phys. B324, 581 (1989).

[5] S. Rychkov, EPFL lectures on conformal field theory in $D \geq 3$ dimensions, arXiv:1601.05000.

[6] D. Simmons-Duffin, The conformal bootstrap, arXiv: 1602.07982 .

[7] P. Kraus and A. Maloney, A cardy formula for three-point coefficients or how the black hole got its spots, J. High Energy Phys. 05 (2017) 160.

[8] J. Cardy, A. Maloney, and H. Maxfield, A new handle on three-point coefficients: OPE asymptotics from genus two modular invariance, J. High Energy Phys. 10 (2017) 136.

[9] C. A. Keller, G. Mathys, and I. G. Zadeh, Bootstrapping chiral CFTs at genus two, arXiv:1705.05862.

[10] M. Cho, S. Collier, and X. Yin, Genus two modular bootstrap, arXiv:1705.05865.

[11] D. Das, S. Datta, and S. Pal, Modular crossings, OPE coefficients and black holes, arXiv:1712.01842.

[12] Y. Kusuki, New properties of large- $c$ conformal blocks from recursion relation, arXiv:1804.06171.

[13] S. Hellerman, A universal inequality for CFT and quantum gravity, J. High Energy Phys. 08 (2011) 130.

[14] D. Friedan and C. A. Keller, Constraints on 2d CFT partition functions, J. High Energy Phys. 10 (2013) 180.

[15] T. Hartman, C. A. Keller, and B. Stoica, Universal spectrum of $2 \mathrm{~d}$ conformal field theory in the large $c$ limit, J. High Energy Phys. 09 (2014) 118.

[16] C. M. Chang and Y. H. Lin, Bootstrapping 2D CFTs in the semiclassical limit, J. High Energy Phys. 08 (2016) 056.

[17] C. M. Chang and Y.H. Lin, Bootstrap, universality and horizons, J. High Energy Phys. 10 (2016) 068.

[18] S. Collier, Y.H. Lin, and X. Yin, Modular bootstrap revisited, arXiv:1608.06241.

[19] S. Collier, P. Kravchuk, Y.H. Lin, and X. Yin, Bootstrapping the spectral function: On the uniqueness of Liouville and the universality of BTZ, arXiv:1702.00423.

[20] M. Cho, S. Collier, and X. Yin, Recursive representations of arbitrary Virasoro conformal blocks, arXiv:1703.09805.

[21] G. W. Moore and N. Seiberg, Polynomial equations for rational conformal field theories, Phys. Lett. B 212, 451 (1988).
[22] H. Sonoda, Sewing conformal field theories. 2., Nucl. Phys. B311, 417 (1988).

[23] P. Kraus, A. Sivaramakrishnan, and R. Snively, Black holes from CFT: Universality of correlators at large $c$, J. High Energy Phys. 08 (2017) 084.

[24] M. Srednicki, The approach to thermal equilibrium in quantized chaotic systems, J. Phys. A 32, 1163 (1999).

[25] L. D'Alessio, Y. Kafri, A. Polkovnikov, and M. Rigol, From quantum chaos and eigenstate thermalization to statistical mechanics and thermodynamics, Adv. Phys. 65, 239 (2016).

[26] R. Mondaini and M. Rigol, Eigenstate thermalization in the two-dimensional transverse field Ising model: II. Offdiagonal matrix elements of observables, Phys. Rev. E 96, 012157 (2017).

[27] A. L. Fitzpatrick, J. Kaplan, and M. T. Walters, Universality of long-distance AdS physics from the CFT bootstrap, J. High Energy Phys. 08 (2014) 145.

[28] N. Lashkari, A. Dymarsky, and H. Liu, Eigenstate thermalization hypothesis in conformal field theory, J. Stat. Mech. (2018) 033101.

[29] A. Dymarsky, N. Lashkari, and H. Liu, Subsystem ETH, Phys. Rev. E 97, 012140 (2018).

[30] S. He, F. L. Lin, and J.j. Zhang, Subsystem eigenstate thermalization hypothesis for entanglement entropy in CFT, J. High Energy Phys. 08 (2017) 126.

[31] P. Basu, D. Das, S. Datta, and S. Pal, Thermality of eigenstates in conformal field theories, Phys. Rev. E 96, 022149 (2017).

[32] N. Lashkari, A. Dymarsky, and H. Liu, Universality of quantum information in chaotic CFTs, J. High Energy Phys. 03 (2018) 070.

[33] T. Faulkner and H. Wang, Probing beyond ETH at large $c$, arXiv: 1712.03464.

[34] A. Dymarsky, Bound on eigenstate thermalization from transport, arXiv:1804.08626.

[35] T. Takayanagi, Holographic Dual of BCFT, Phys. Rev. Lett. 107, 101602 (2011).

[36] M. Fujita, T. Takayanagi, and E. Tonni, Aspects of AdS/BCFT, J. High Energy Phys. 11, 043 (2011).

[37] Y. Kusuki and T. Takayanagi, Renyi entropy for local quenches in 2D CFT from numerical conformal blocks, J. High Energy Phys. 01 (2018) 115.

[38] E. M. Brehm, D. Das, and S. Datta, Probing thermality beyond the diagonal, arXiv:1804.07924.

[39] A. Romero-Bermdez, P. Sabella-Garnier, and K. Schalm, A Cardy formula for off-diagonal three-point coefficients; or, how the geometry behind the horizon gets disentangled, arXiv:1804.08899. 